\title{
Extracellular Matrix Mediates BMP-2 in a Model of Temporomandibular Joint Osteoarthritis
}

\author{
Maya Shirakura ${ }^{a, c}$ Vardit Kram $^{a}$ Jennifer Robinson ${ }^{b}$ Sheena Sikka ${ }^{a}$ \\ Tina M. Kilts ${ }^{a}$ Sunil Wadhwa ${ }^{b}$ Marian F. Young ${ }^{a}$ \\ ${ }^{a}$ NIDCR, National Institutes of Health, Bethesda, MD, and ${ }^{b}$ Columbia University, New York, NY, USA; \\ 'Hiroshima University, Hiroshima, Japan
}

\section{Keywords}

Biglycan · Fibromodulin · Mineralization - Growth factors ·

Turnover · Osteoclasts

\begin{abstract}
Temporomandibular joint (TMJ) osteoarthritis (OA) is a complex disease that affects both cartilage and subchondral bone. It is accompanied by loss of extracellular matrix (ECM) and may be controlled by bone morphogenetic protein-2 (BMP-2). We analyzed the effect of BMP-2 in both cartilage and subchondral bone in a TMJ-OA animal model that is deficient in biglycan (Bgn) and fibromodulin (Fmod) $\left(\mathrm{Bgn}^{-/-}\right.$ $\mathrm{Fmod}^{-/-}$). Whole mandibles were dissected from 3-week-old wild-type (WT) and $\mathrm{Bgn}^{-/-} \mathrm{Fmod}^{-/-}$mice and incubated with and without $250 \mu \mathrm{g} / \mathrm{mL}$ BMP-2 for 2 days using an explant culture system. Condyle growth was measured by microCT and the expression levels of cartilage and bone-related genes were analyzed using RT-PCR or by immunohistochemistry from condyles that contained an intact cartilage/ subchondral bone interface. Osteoclast activity was estimated by tartrate-resistant acid phosphatase (TRAP) staining and by TRAP, Rankl, and Adamts 4 mRNA expression levels. Our results showed that most parameters examined were slightly up-regulated in WT samples treated with
\end{abstract}

\section{KARGER}

(C) 2017 S. Karger AG, Basel

E-Mail karger@karger.com

www.karger.com/cto
BMP-2, and this up-regulation was significantly enhanced in the $\mathrm{Bgn}^{-/-} \mathrm{Fmod}^{-/-}$mice. The up-regulation of both catabolic and anabolic agents did not appear to positively affect the overall growth of $\mathrm{Bgn}^{-/-} \mathrm{Fmod}^{-/-}$condyles compared to WT controls. In summary, the up-regulation of both anabolic and catabolic genes in the WT and $\mathrm{Bgn}^{-/-} \mathrm{Fmod}^{-/-}$TMJs treated with BMP-2 suggests that BMP increases matrix turnover in the condyle, and, further, that Bgn and Fmod could have protective roles in regulating this process.

(c) 2017 S. Karger AG, Basel

\section{Abbreviations used in this paper}

Acan aggrecan

Bgn biglycan

BMP-2 bone morphogenetic protein-2

ECM extracellular matrix

Fmod fibromodulin

IHC immunohistochemistry

OA osteoarthritis

SLRPs small leucine-rich proteoglycans

TMJ temporomandibular joint

TRAP tartrate-resistant acid phosphatase

WT wild type 


\section{Introduction}

Temporomandibular joint (TMJ) disorders afflict approximately $5-10 \%$ of the US population [Liu and Steinkeler, 2013]. These disorders encompass a collection of at least 12 clinical phenotypes, one of which is degenerative TMJ disease [Schiffman et al., 2014]. Individuals with this degenerative joint disease experience irreversible damage to the joint complex, including degradation and loss of extracellular matrix (ECM) proteins necessary for joint function. Unfortunately, in most cases, this irreversible damage occurs before symptoms present and the disease is diagnosed [Wang et al., 2015]. Therefore, efforts to identify markers and molecular mechanisms characteristic of degenerative TMJ disease in the early phases are needed to detect and develop treatments that can prevent the irreversible process of the disease.

The TMJ is a unique articulating joint comprised of the mandibular condyle which fits into the mandibular fossa of the temporal bone. The articular disk and the articular cartilage of the condyle are both fibrocartilaginous tissues that cover these two bones and promote articulation. The fibrocartilage of the mandibular condyle is comprised of ECM proteins and proteoglycans that provide the necessary properties to support the loads on the condyle during mastication and verbal communication. Within this ECM, there exists small leucine-rich proteoglycans (SLRPs) including biglycan (Bgn) and fibromodulin (Fmod), which play a role in sequestration of important signaling molecules and regulating collagen fibrillogenesis [Ameye and Young, 2002]. Specifically, Bgn and Fmod modulate TGF super family signaling activity, including bone morphogenetic protein (BMP) signaling [Wadhwa et al., 2004; Young et al., 2006]. BMP signaling has well-established roles in chondrogenesis and endochondral bone formation [Long and Ornitz, 2013]. Abnormal activation of this signaling causes heterotopic cartilage and bone formation [Billings et al., 2008; Huegel et al., 2015]. Specifically, excessive BMP-2 signaling has recently been shown to be involved in heterotopic cartilage formation and the pathogenesis of TMJ osteoarthritis (OA) [Albilia et al., 2013; Bechtold et al., 2016]. However, the mechanism of excessive BMP-2 signaling on the mandibular condylar cartilage and its implications in TMJOA are unknown.

We have previously developed a murine model with accelerated TMJ-OA in mice double-deficient in Bgn and Fmod $\left(\mathrm{Bgn}^{-/-} \mathrm{Fmod}^{-/-}\right)$. Using this model, we determined that $\mathrm{Bgn}^{-/-} \mathrm{Fmod}^{-/-}$mice develop TMJ-OA at 6-9 months of age whereas WT mice develop TMJ-OA at 12-18

ECM-Mediated Control of BMP-2 in the TMJ months of age [Wadhwa et al., 2005a, 2005b; Chen et al., 2009]. Consequently, the goal of this study was to examine BMP-2 signaling in $\mathrm{Bgn}^{-/-} \mathrm{Fmod}^{-/-}$mice. We hypothesized that BMP-2 treatment would promote overactivation of chondrogenic markers in the absence of Bgn and Fmod compared to WT mice due to dysregulation of the signaling pathway. Greater understanding of the early signaling events that mediate TMJ-OA is critical in the development of diagnostic tools and interventional therapeutics.

\section{Materials and Methods}

Animals

Female $\mathrm{Bgn}^{-/-} \mathrm{Fmod}^{-/-}$mice and their strain-matched wild-type (WT) counterparts $(\mathrm{C} 57 \mathrm{BL} / 6)$ were used with approval from the Animal Care and Use Committee, National Institutes of Health (NIDCR-12-655). The generation and genotyping of the $\mathrm{Bgn}^{-/-} \mathrm{Fmod}^{-/-}$have been reported previously [Wadhwa et al., 2005a].

Mandibular ex vivo Organ Culture and Treatment with BMP-2 Mandibular condyles were harvested from 3-week-old WT or $\mathrm{Bgn}^{-/-} \mathrm{Fmod}^{-/-}$mice (for histology) or 5-week-old mice (for mRNA), dissected in half, and cultured in 24-well plates in DMEM and Pen/Strep antibiotics supplemented with $100 \mathrm{nmol} / \mathrm{L}$ ascorbic acid for $24 \mathrm{~h}$. The condyles were then incubated with or without $250 \mathrm{ng} / \mathrm{mL}$ human carrier-free recombinant BMP-2 (eBioscience). The left mandibular condyle was treated with BMP-2, and the right was treated with vehicle control. After 2 days, the explant cultures were collected and prepared for histology and quantitative RTPCR (online suppl. Fig. S1; for all online suppl. material, see www.karger.com/doi/10.1159/000464102). For each sample, 5 condyles were combined, and each experiment was repeated at least 3 times.

\section{MicroCT}

Treated mandibles were fixed and scanned using a $\mu$ CT 50 (Scanco Medical AG, Bassersdorf, Switzerland). Scans were performed at $70 \mathrm{kV}, 85 \mu \mathrm{A}, 300-\mathrm{ms}$ integration time, and at a resolution of $10 \mu \mathrm{m}$. After reconstruction, the images were stored in $3 \mathrm{D}$ arrays and used to measure the height of the mandible as shown in Figure 1a, b.

\section{Quantitative RT-PCR}

Expression levels of cartilage- and bone-related genes were analyzed by RT-PCR using RNA obtained from condyles that contained both cartilage and subchondral bone. RNA was isolated using TRIzol (Invitrogen) and purified with RNeasy. cDNA was generated by reverse transcribing total RNA using iScript from BioRad and subjecting it to RT-PCR using SYBER Master Mix (Applied Biosystem) in a BioRad MyiQ quantitative PCR thermocycler. Each cDNA (5-10 ng) was amplified using the primers shown in online supplementary Table S1. Gene expression was normalized to the housekeeping gene, S29. For each sample, 5 condyles were combined, and each experiment was repeated at least 3 times. 
Fig. 1. a Representative microCT image of a 3-week-old mouse condyle after explant culture indicating the growth regions that were measured. b Height of the condyles in WT or $\mathrm{Bgn}^{-/-} \mathrm{Fmod}^{-/-}$tissue with or without BMP-treatment. ${ }^{*} p<0.05$.

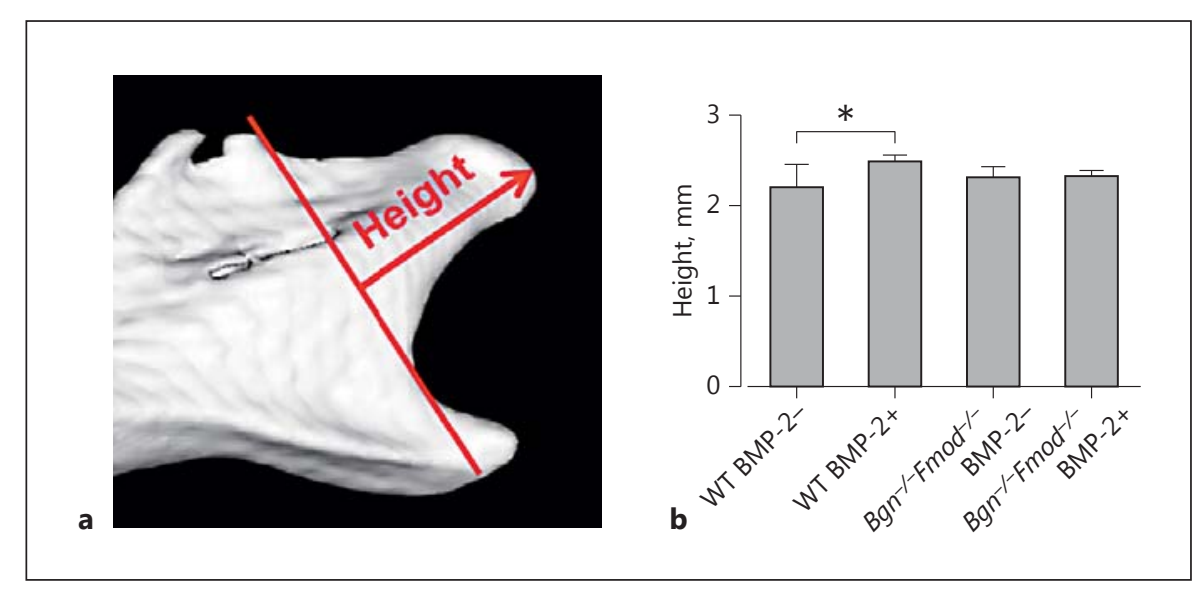

\section{Histology, Staining, and Quantification}

Paraffin-embedded sections were dewaxed in xylene washes and rehydrated through graded ethanol series (100, 95, and $75 \%$ and water). Tissue sections were stained with hematoxylin and eosin or safranin O. For immunohistochemistry (IHC), rehydrated sections were enzymatically treated with ABCase and incubated with primary antibodies at $4{ }^{\circ} \mathrm{C}$ overnight including polyclonal rabbit antimouse aggrecan (Acan; $1: 100=5 \mathrm{mg} / \mathrm{mL}$ rabbit IgG catalog No. AB1031; Abcam) and polyclonal rabbit antimouse type I collagen $(1: 2,000$, rabbit antiserum, LF-68, a gift from Dr. Larry Fisher). The broad-spectrum immunoperoxidase AEC kit (Picture Plus; Zymed) was subsequently used to detect the immunoreactivity according to the manufacturer's instructions. The sections were counterstained with hematoxylin. Images of the stained sections were captured, and the area of positive staining accessed using software from Image-Pro. Nonimmune immunoglobulins of the same isotype as the primary antibody served as negative controls, and showed no positive staining (not shown). Safranin O-positive staining in the treated TMJs was also accessed using Image-Pro software (online suppl. Fig. S2). Osteoclast formation was estimated by counting the number of tartrate-resistant acid phosphatase (TRAP; 387A Sigma)-positive multinucleated cells in 5-7 sections/ sample.

\section{Statistical Analysis}

Values are presented as means \pm SD. Statistical significance of differences among means was determined by two-way analysis of variance with post hoc analysis by the Bonferroni method using SPSS. Statistical significance was defined as $p<0.05$.

\section{Results}

The experimental strategy for examining BMP-2 function in ex vivo explants is outlined in online supplementary Figure S1. Briefly, hemi-mandibles were dissected from the heads of WT and $\mathrm{Bgn}^{-/-} \mathrm{Fmod}^{-/-}$mice and included the entire condyle with its subchondral bone.
Samples from 3-week-old mice were used for histology while 5-week-old mice were used for mRNA extraction. The hemi-mandibles were then cultured in the presence or absence of BMP-2 for $48 \mathrm{~h}$ and processed for microCT, histology, and mRNA isolation for RT-PCR.

Hemi-mandibles from WT and $\mathrm{Bgn}^{-/-} \mathrm{Fmod}^{-/-}$mice incubated with or without BMP-2 were first analyzed by microCT to determine the relative height of the condyle after treatment (Fig. 1a). This analysis showed that WT condyles responded with a slight but significant increase in length (Fig. 1b), but the $\mathrm{Bgn}^{-/-} \mathrm{Fmod}^{-/-}$condyles did not. This finding prompted us to further examine the underlying cell and molecular foundation by histology, IHC, and quantitative RT-PCR.

At 3 weeks of age, the TMJ is composed of a thick fibrocartilage surface that covers a layer of cartilage that can be clearly visualized by staining with safranin $\mathrm{O}$ (online suppl. Fig. S2). The cartilage layer lies on top of the subchondral bone that composes the majority of the condyle structure. The area of safranin O-positive staining in WT mandibles treated with BMP-2 was unchanged after the 48-h BMP treatment. However, the area of safranin $\mathrm{O}$ in condyles from $\mathrm{Bgn}^{-/-} \mathrm{Fmod}^{-/-}$mice was significantly increased (Fig. 2a, b). To confirm these observations, we next stained the sections for the expression of Acan, a known marker of differentiated cartilage tissue. This showed that BMP-2 slightly increased the extent of Acan staining in WT tissue and more so in the $\mathrm{Bgn}^{-/-} \mathrm{Fmod}^{-/-}$condyles (Fig. 2c, d). When the relative expression of mRNA for Acan was examined with BMP2 treatment, we found that both WT and $\mathrm{Bgn}^{-/-} \mathrm{Fmod}^{-/-}$ had increased Acan levels, but that in the $\mathrm{Bgn}^{-/} \mathrm{Fmod}^{-/-}$ the BMP-2 response was much more robust that in the WT (Fig. 2e).
86

Cells Tissues Organs 2017;204:84-92 DOI: $10.1159 / 000464102$
Shirakura/Kram/Robinson/Sikka/Kilts/ Wadhwa/Young 


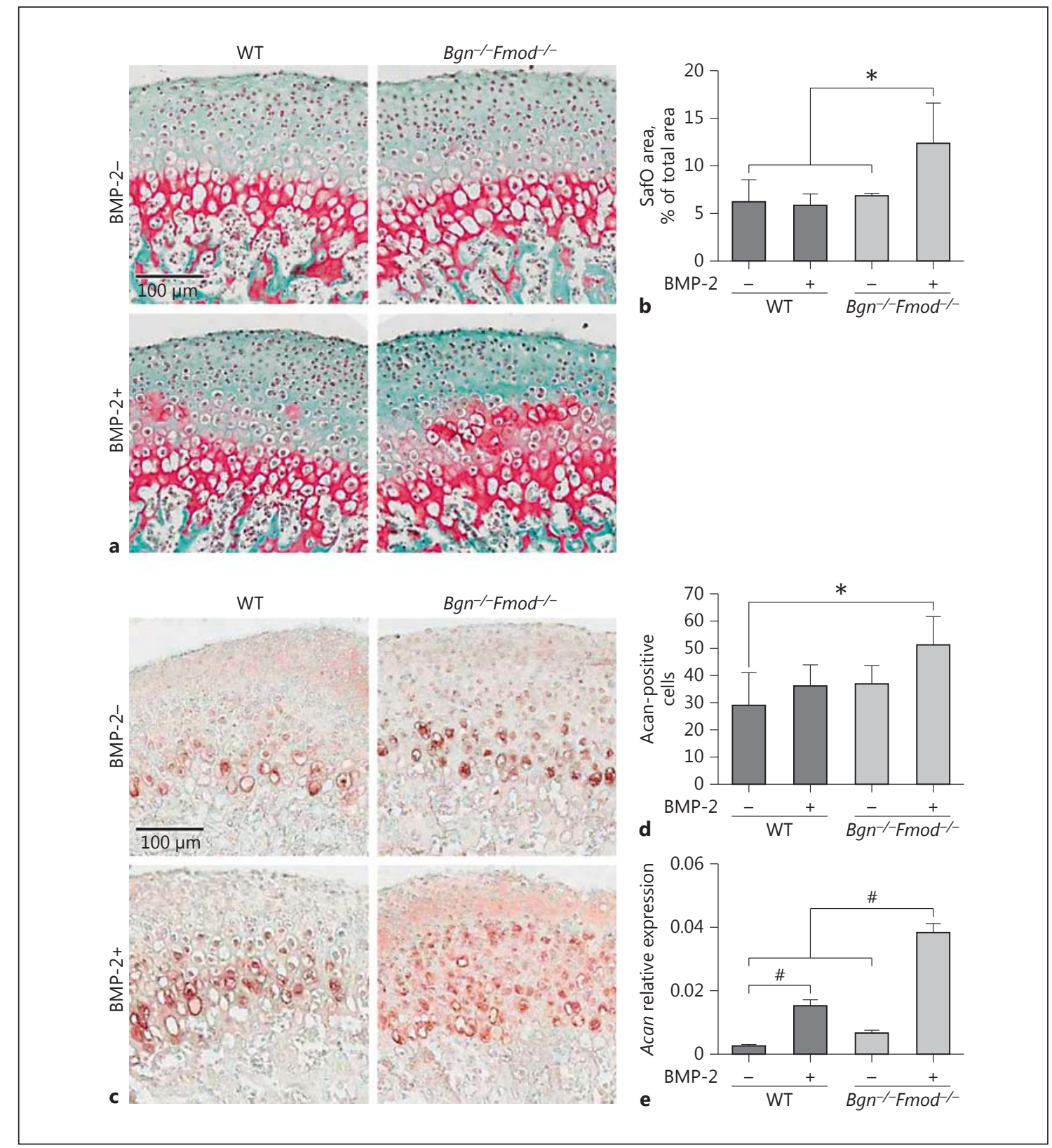

Fig. 2. a Safranin O staining of WT (left panels) and $\mathrm{Bgh}^{-/-} \mathrm{Fmod}^{-/-}$condyles (right panels) treated without (top panels) or with (bottom panels) BMP-2. b Histomorphometic quantitation of safranin O (SafO)-positive area/ total area. c IHC for Acan in WT (left panels) and $\mathrm{Bgn}^{-/-} \mathrm{Fmod}^{-/-}$condyles (right panels) treated without (top panel) or with (bottom panels) BMP-2. d Histomorphic evaluation of Acan-positive cells. e Relative expression of Acan mRNA in WT versus $\mathrm{Bgn}^{-l-} \mathrm{Fmod}^{-1-}$ with or without BMP-2. ${ }^{*} p<0.05,{ }^{\#} p<0.01$.

To deepen our understanding of the molecular basis for the observed changes that we saw in cartilage tissue morphology, the mRNA expression levels for additional key cartilage genes were determined. This analysis showed that BMP-2 increased the levels of type II colla- gen (Col2; Fig. 3a) and type X collagen (Col10; Fig. 3b), Sox9, the master gene known to regulate cartilage formation (Fig. 3c), and the chondrocyte controlling gene $I \mathrm{hh}$ (Fig. 3d), and that the relative level of induction was much greater in the $\mathrm{Bgn}^{-/-} \mathrm{Fmod}^{-/-}$condyles. Taken to- 
Fig. 3. RT-PCR of cartilage-expressed genes in WT and $\mathrm{Bgn}^{-/} \mathrm{Fmod}^{-/-}$treated without or with BMP-2. a Type II collagen (Col2). b Type X collagen (Col10). c Sox9. d Ihh. ${ }^{*} p<0.05,{ }^{*} p<0.01$.

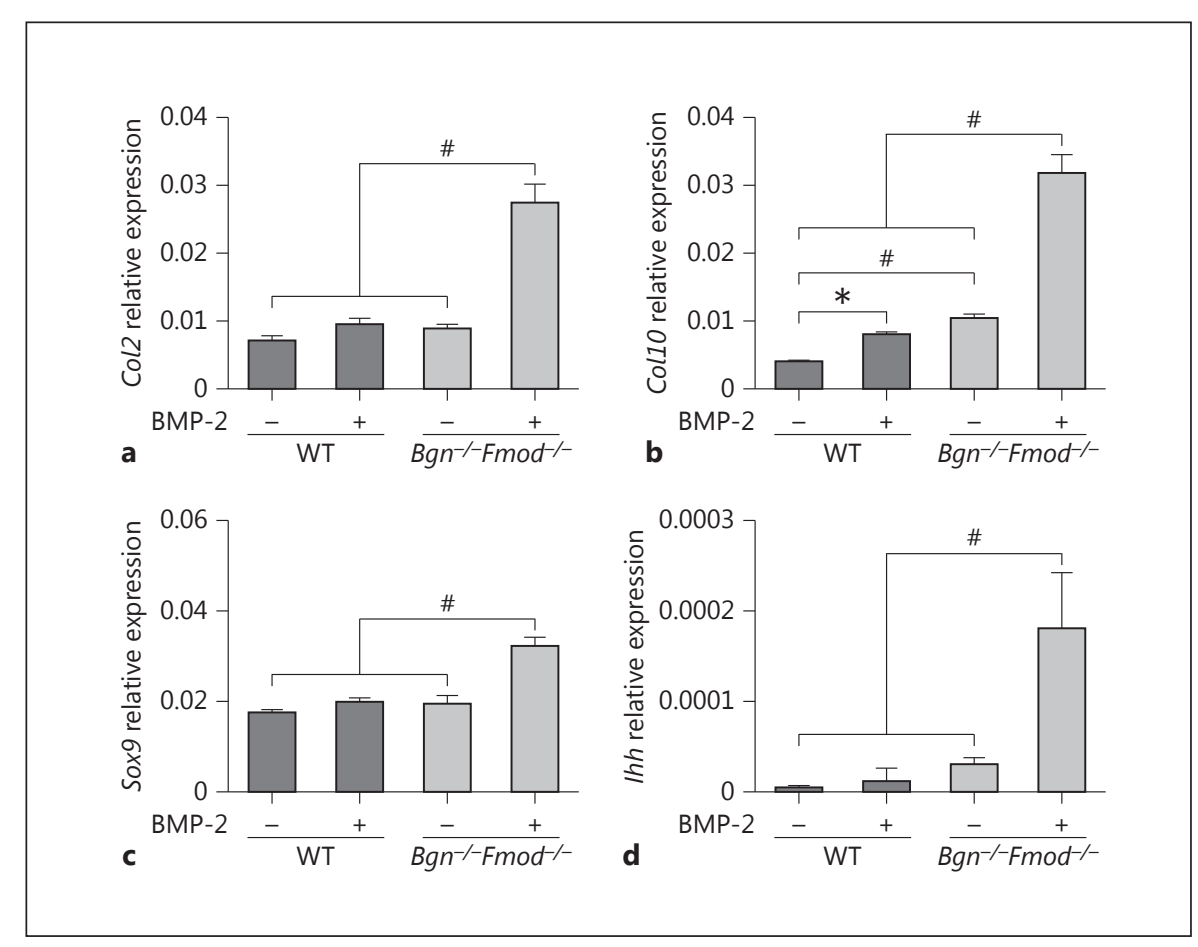

gether, these data showed that the $\mathrm{Bgn}^{-/-} \mathrm{Fmod}^{-/-}$condyles are more sensitive to the effects of BMP-2 causing expansion of the cartilage zone resulting in increased levels of safranin $\mathrm{O}$, Acan-positive tissue, as well in the expression of the cartilage-related genes Col2, Col10, Sox9, and $I h h$.

We next examined the effects of BMP-2 on the proteins and mRNAs that are made by the surrounding fibrocartilage and bone. This analysis showed that the extent of type I collagen (Col1) in the fibrocartilage was slightly increased (but not significantly) in WT condyles treated with BMP-2, and that the $\mathrm{Bgn}^{-/-} \mathrm{Fmod}^{-/-}$condyles were much more responsive (Fig. 4a). RT-PCR of mRNA encoding Col1 mRNA showed a parallel significant increase in type I collagen in the $\mathrm{Bgn}^{-/-} \mathrm{Fmod}^{-/-}$condyles treated with BMP-2 compared with similarly treated WT explants (Fig. 4b). The master gene, Runx2, known to regulate many bone-enriched genes, including type I collagen, was found to be regulated in the same manner as type I collagen (Fig. 4c). Osterix (Osx), the master regulatory factor operating downstream of Runx2, showed a different pattern compared to Runx2 whereby induction by BMP- 2 in WT condyles was almost (but not completely) at the same level as the $\mathrm{Bgn}^{-/-} \mathrm{Fmod}^{-/-}$condyles (Fig. 4d). A downstream target of Wnt called Wisp1/ Ccn4, which is known to control bone turnover, was also examined and showed constitutive elevation in WT versus $\mathrm{Bgn}^{-/-} \mathrm{Fmod}^{-/-}$, and that this increased expression was further enhanced by treatment with BMP-2 (Fig. 4e).

The enhancement of genes known to regulate bone and cartilage formation led us to question whether skeletal turnover in the $\mathrm{Bgn}^{-/-} \mathrm{Fmod}^{-/-}$condyles was differentially affected by BMP-2. To test this possibility, WT and $\mathrm{Bgn}^{-/-} \mathrm{Fmod}^{-/-}$condyles were treated with BMP-2 and stained for TRAP to identify osteoclasts. This analysis showed that the number of multinucleated TRAP-positive cells was not significantly changed in BMP-2-treated WT condyles; in contrast, $\mathrm{Bgn}^{-/-} \mathrm{Fmod}^{-/-}$condyles showed a higher number of TRAP-positive cells that was increased with BMP-2 (Fig. 5a). Analysis of mRNA for TRAP (Acp5) showed a similar outcome where BMP2-treated $\mathrm{Bgn}^{-/-} \mathrm{Fmod}^{-/-}$condyles had much greater expression than BMP-2-treated WT samples (Fig. 5b). Interestingly, untreated $\mathrm{Bgn}^{-/-} \mathrm{Fmod}^{-/-}$condyles also had elevated TRAP mRNA expression compared to untreated WT condyles. Analysis of additional catabolic mRNAs, Rankl (Fig. 5c) and Adamts4 (Fig. 5d), showed a similar pattern to TRAP mRNA where the $\mathrm{Bgn}^{-/ 0} \mathrm{Fmod}^{-/-}$condyles had constitutively higher levels than WT condyles. BMP-2 treatment of $\mathrm{Bgn}^{-/} \mathrm{Fmod}^{-/-}$condyles further increased Rankl and Adamts mRNA compared to WT controls.
Shirakura/Kram/Robinson/Sikka/Kilts/ Wadhwa/Young 


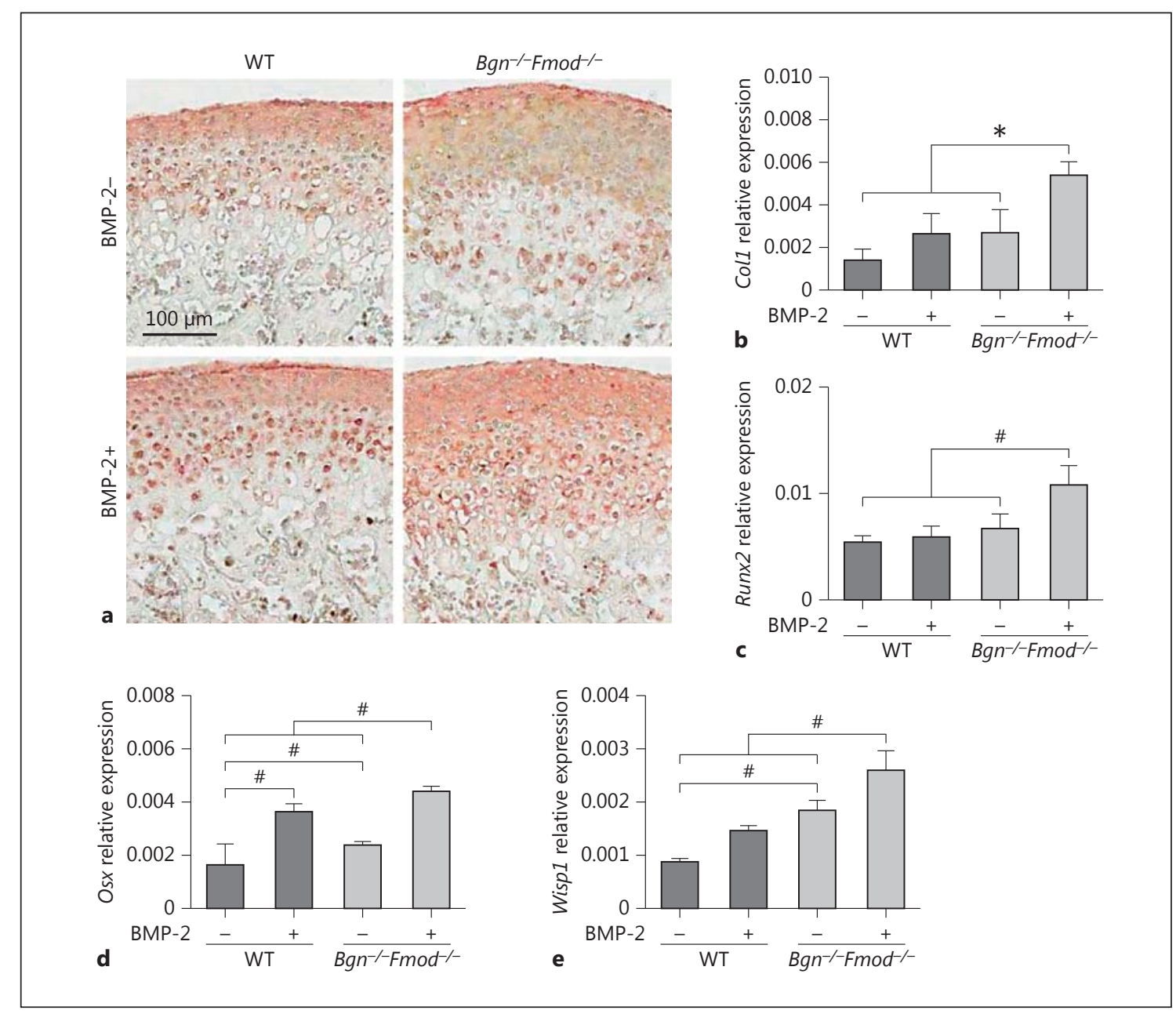

Fig. 4. IHC and RT-PCR of genes expressed in fibrocartilage and bone. a IHC of type I collagen in WT (left panels) and $\mathrm{Bgn}^{-/-} \mathrm{Fmod}^{-1-}$ condyles (right panels) treated without (top panels) or with (bottom panels) BMP2. b-e RT-PCR of type I collagen (Col1) (b), Runx2 (c), osterix (Osx) (d), and Wisp1 mRNA (e) in WT and $\mathrm{Bgn}^{-/-} \mathrm{Fmod}^{-/-}$condyles treated with or without BMP-2. ${ }^{*} \mathrm{p}<0.05,{ }^{*} p<0.01$.

\section{Discussion}

For patients who experience TMJ-OA, irreversible degeneration of the joint often occurs prior to detection or diagnosis. Thus, early detection and diagnosis with biomarkers could lead to early intervention and reduce the incidence of irreversible damage to the TMJ. The goal of this study was to determine whether excessive BMP-2 signaling results in overactivation of chondrogenesis in a $\mathrm{Bgn}^{-/-} \mathrm{Fmod}^{-/-}$model of TMJ-OA. Results from this study illustrate that BMP-2 treatment potentiated the induction of Acan, Col2, Col 10, and Ihh in $\mathrm{Bgn}^{-/-} \mathrm{Fmod}^{-/-}$mice. Excess BMP-2 signaling has also been implicated in the pathogenesis of accelerated TMJ-OA in Pgr4-knockout

ECM-Mediated Control of BMP-2 in the TMJ
(KO) mice [Bechtold et al., 2016]. In contrast, absence of BMP signaling in transgenic mice with Bmp1Ra conditionally deleted in neural crest cells led to failure of articular disk separation from the hypoplastic condyle and underdevelopment of the mandibular condylar cartilage [Gu et al., 2014]. Taken together, the results suggest that a threshold of BMP-2 is needed for normal TMJ development.

The mandibular condylar cartilage is unique in that it is derived from the periosteum and undergoes endochondral ossification. In other growth plates, it is believed that there are a finite number of progenitor cells, and once these cells are depleted, growth stops [Hinton et al., 2015]. A similar mechanism may occur in the TMJ. In mice, for 


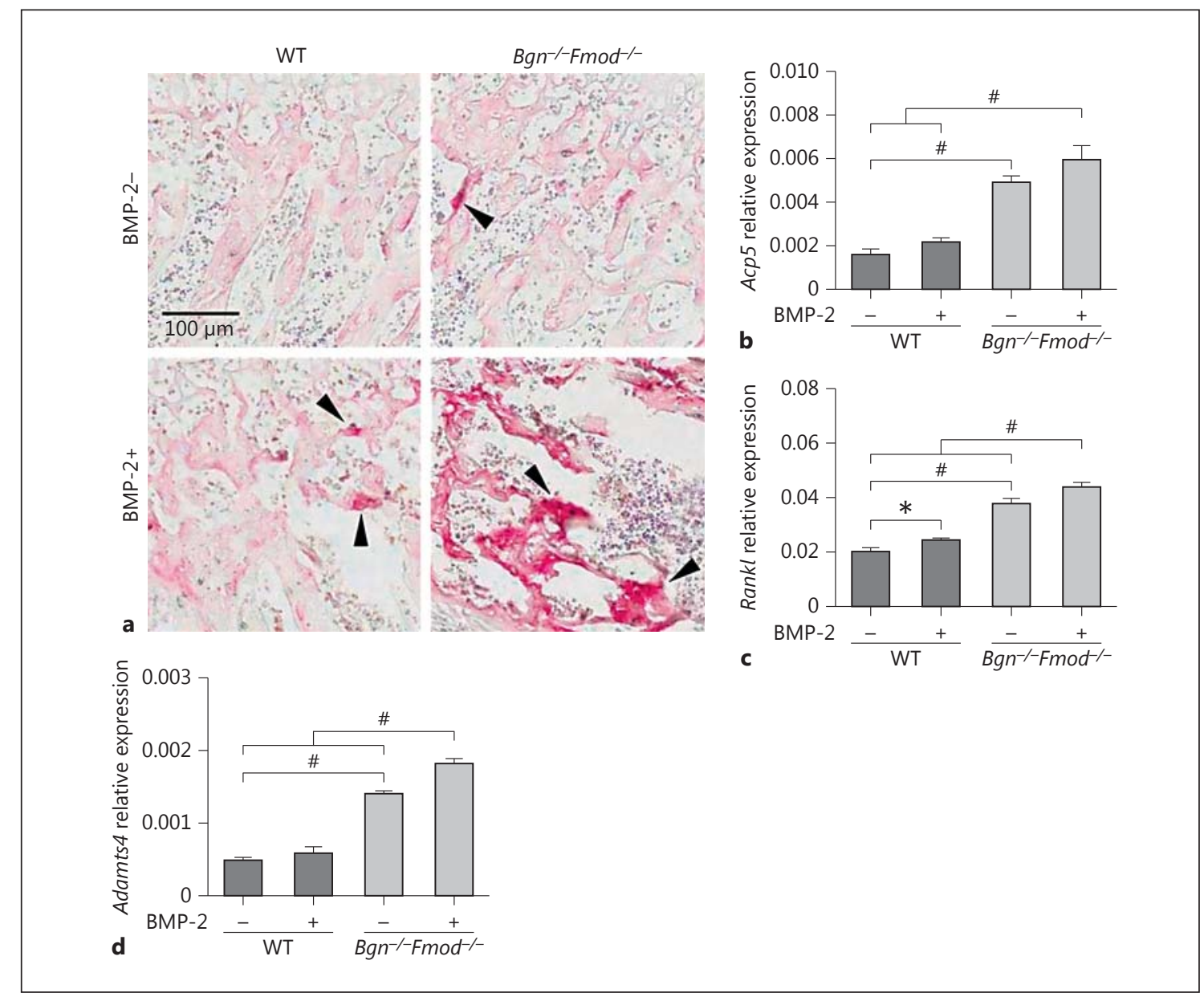

Fig. 5. Assessment of bone resorption. a Tartrate-resistant acid phosphatase (TRAP) staining of osteoclasts in WT (left panels) and $\mathrm{Bgn}^{-/} \mathrm{Fmod}^{-/-}$condyles (right panels) treated without (top panels) or with (bottom panels) BMP-2. RT-PCR to measure levels of TRAP (Acp5) (b), Rankl (c), and Adamts4 mRNA (d).

example, the mandibular condylar cartilage rapidly undergoes endochondral ossification until 2 months of age [Ghassemi et al., 2017]. Afterwards, the mandibular condylar cartilage becomes senescent and phenotypically resembles other articular cartilages [Livne et al., 1985]. Therefore, in the absence of Bgn and Fmod, excessive BMP-2 signaling may cause accelerated progenitor cell depletion resulting in accelerated age-related TMJ degeneration. Future studies will focus on delivering BMP-2 inhibitors to young (growing) and adult (nongrowing) mice to examine if they can inhibit age-related TMJ-OA in the $\mathrm{Bgn}^{-/-} \mathrm{Fmod}^{-/-}$mice in order to further test this hypothesis.

It is well known that Acan is a major proteoglycan in cartilage and that it has important roles in regulating cartilage integrity. In the present study, we focused on Bgn and Fmod because we found they are both abundantly expressed and localized in the articular cartilage of the mandibular condyle [Wadhwa et al., 2005a]. Interestingly, in the $\mathrm{Bgn}^{-/-} \mathrm{Fmod}^{-/-}$condyles, the expression of Acan is first increased at 3 and 5 weeks of age, and it decreased with further reduction at 24 weeks of age compared to WT condyles. We believe this is due, in part, to overactive TGF- $\beta$ production leading to an up-regulation of proteolytic enzymes that degrade Acan including Adamts 4 and Adamts5 [Embree et al., 2010]. In this context, we cannot exclude the possibility that some of the effects we see in the BMP-2 responsiveness in the $\mathrm{Bgn}^{-/-} \mathrm{Fmod}^{-/-}$mice could, in fact, be from alterations in Acan levels and function.

In our previous study, we found that TGF- $\beta$ signaling was elevated in $\mathrm{Bgn}^{-/-} \mathrm{Fmod}^{-/-}$mice. Our results with 
BMP-2 are similar; however, in this study, we found that BMP-2 also caused an increase in Rankl [Embree et al., 2010]. Increased Rankl in the $\mathrm{Bgn}^{-/-} \mathrm{Fmod}^{-/-}$mice was confirmed by our previously published microarray data [Embree et al., 2011], suggesting that osteoclastogenesis may be specific for the BMP-2 signaling pathway rather than the TGF- $\beta$ signaling pathway.

Bgn and Fmod have a highly ordered structure that is composed of a core protein with extensive posttranslational modifications, including the addition of chondroitin/dermatan sulfate (Bgn) or keratin sulfate glycosaminoglycan (Fmod) chains. Currently, it is not known what region of these small proteoglycans is important for the functions we show they have in the present study. An important next step will be to test the role of the core protein compared to the proteoglycan form (with glycosaminoglycan chains) to see what part of these SLRP molecules is biologically active. In this regard, it is interesting to note that 3 different families with point mutations in the core protein of Bgn have bone shortening suggesting the overall importance of the core protein in Bgn function [Cho et al., 2016]. Clearly, more experiments are needed to fully understand the SLRP mechanism of action requiring comprehensive and systematic testing of all the structural parts of Bgn and Fmod either by gain-of-function or "knockin" genetic manipulation.

Considering how many SLRPs populate skeletal tissues, it is likely that there is some overlap in their functions including in the ability to bind and regulate growth factors such as the TGF- $\beta$ family members. When we examined mice single deficient in either decorin or Fmod, we did not see any major bone phenotype and so did not pursue the single-decorin KO or single-Fmod KO further in this context. With regard to cartilage, both the singleBgn KO and the single-Fmod KO display mild OA, and because Fmod and Bgn are so highly coexpressed in the cartilage of the condyle, we suspected they had overlapping and possibly compensatory functions there. By making mice deficient in both Fmod and Bgn, we subsequent- ly uncovered new roles for these SLRPS that were masked by a compensation effect. This was evident in the $\mathrm{Bgn}^{-/-} \mathrm{Fmod}^{-/-}$, which had much earlier and severe OA than the single-Bgn- and single-Fmod-deficient mice [Ameye et al., 2002]. Because of the nature of the genetic model used, we cannot tell whether Bgn or Fmod dominates in the functions we discovered, namely SLRP control of BMP-2-induced bone and cartilage turnover.

As mentioned earlier, Bgn and Fmod are members of the SLRPs. Members of this family have been linked to OA by their action in mediating extracellular collagen organization, mediating TGF- $\beta$ signaling, and/or regulating subchondral bone turnover (for review see, $\mathrm{Ni}$ et al. [2014]). Therefore, decreased expression of Bgn and Fmod may be a biomarker for the early stages of TMJ-OA and/or potentially indicate a higher probability of developing TMJ-OA.

The detection of TMJ-OA usually occurs after there is irreversible damage to the joint. Therefore, efforts to detect the disease earlier and thus to prevent TMJ-OA are needed. In our model, there is excessive BMP-2 signaling. Therefore, monitoring the expression of BMP-2, Bgn, and Fmod may help in the early detection of TMJ-OA. Furthermore, manipulating the expression of these proteins may be beneficial in preventing the progression of the disease process.

\section{Acknowledgments}

We would like to thank A. Donald and L. Li for technical assistance. This work was supported in part by the intramural program of the National Institute of Dental and Craniofacial Research (V.K., S.S., T.M.K, and M.F.Y.) and by a fellowship from the Japanese Promotion for the Advancement of Science (M.S.).

\section{Disclosure Statement}

There are no conflicts of interest to disclose.

\section{References}

ECM-Mediated Control of BMP-2 in the TMJ
Albilia, J.B., H.C. Tenenbaum, C.M. Clokie, et al (2013) Serum levels of BMP-2, 4, 7 and AHSG in patients with degenerative joint disease requiring total arthroplasty of the hip and temporomandibular joints. J Orthop Res 31: 44-52.

Ameye, L., M.F. Young (2002) Mice deficient in small leucine-rich proteoglycans: novel in vivo models for osteoporosis, osteoarthritis, Ehlers-Danlos syndrome, muscular dystrophy, and corneal diseases. Glycobiology 12: 107r-116r.
Bechtold, T.E., C. Saunders, C. Mundy, et al (2016) Excess BMP signaling in heterotopic cartilage forming in Prg4-null TMJ discs. J Dent Res 95: 292-301.

Billings, P.C., J.L. Fiori, J.L Bentwood, et al (2008) Dysregulated BMP signaling and enhanced osteogenic differentiation of connective tissue progenitor cells from patients with fibrodysplasia ossificans progressiva (FOP). J Bone Miner Res 23: 305-313. 
Chen, J., T. Gupta, J.A. Barasz, et al (2009) Analysis of microarchitectural changes in a mouse temporomandibular joint osteoarthritis model. Arch Oral Biol 54: 1091-1098.

Cho, S.Y., J.S. Bae, N.K. Kim, et al (2016) BGN mutations in X-linked spondyloepimetaphyseal dysplasia. Am J Hum Genet 98: 12431248.

Embree, M.C., T.M. Kilts, M. Ono, et al (2010) Biglycan and fibromodulin have essential roles in regulating chondrogenesis and extracellular matrix turnover in temporomandibular joint osteoarthritis. Am J Pathol 176: 812-826.

Embree, M., M. Ono, T. Kilts, et al (2011) Role of subchondral bone during early-stage experimental TMJ osteoarthritis. J Dent Res 90: 1331-1338.

Ghassemi Nejad, S., T. Kobezda, I. Tar, Z. Szekanecz (2017) Development of temporomandibular joint arthritis: The use of animal models. Joint Bone Spine 84:145-151.

Gu S, W. Wu, C. Liu, et al (2014) BMPRIA mediated signaling is essential for temporomandibular joint development in mice. PLoS One 29: e101000.

Hinton RJ, Jing J, Feng JQ (2015) Genetic influences on temporomandibular joint development and growth. Curr Top Dev Biol 115: 85-109.
Huegel, J., M. Enomoto-Iwamoto, F. Sgariglia, E. Koyama, M. Pacifici (2015) Heparanase stimulates chondrogenesis and is up-regulated in human ectopic cartilage: a mechanism possibly involved in hereditary multiple exostoses. Am J Pathol 185: 1676-1685.

Liu, F., A. Steinkeler (2013) Epidemiology, diagnosis, and treatment of temporomandibular disorders. Dent Clin North Am 57: 465-479.

Livne, E., K. von der Mark, M. Silbermann (1985) Morphologic and cytochemical changes in maturing and osteoarthritic articular cartilage in the temporomandibular joint of mice. Arthritis Rheum 28: 1027-1038.

Long, F., D.M. Ornitz (2013) Development of the endochondral skeleton. Cold Spring Harb Perspect Biol 5: a008334.

Ni, G.X., Z. Li, Y.Z. Zhou (2014) The role of small leucine-rich proteoglycans in osteoarthritis pathogenesis. Osteoarthritis Cartilage 22: 896-903.
Schiffman, E., R. Ohrbach, E. Truelove, et al (2014) Diagnostic criteria for temporomandibular disorders (DC/TMD) for clinical and research applications: recommendations of the International RDC/TMD Consortium Network and Orofacial Pain Special Interest Group. J Oral Facial Pain Headache 28: 6-27.

Wadhwa, S., M.C. Embree, Y. Bi, M.F. Young (2004) Regulation, regulatory activities, and function of biglycan. Crit Rev Eukaryot Gene Expr 14: 301-315.

Wadhwa, S., M.C. Embree, T.M. Kilts, M.F. Young, L.G. Ameye (2005a) Accelerated osteoarthritis in the temporomandibular joint of biglycan/fibromodulin double-deficient mice. Osteoarthritis Cartilage 13: 817-827.

Wadhwa, S., M. Embree, L. Ameye, M.F. Young (2005b) Mice deficient in biglycan and fibromodulin as a model for temporomandibular joint osteoarthritis. Cells Tissues Organs 181: 136-143.

Wang, X.D., J.N. Zhang, Y.H. Gan, Y.H. Zhou (2015) Current understanding of pathogenesis and treatment of TMJ osteoarthritis. J Dent Res 94: 666-673.

Young, M.F., Y. Bi, L. Ameye, et al (2006) Small leucine-rich proteoglycans in the aging skeleton. J Musculoskelet Neuronal Interact 6: 364-365. 